\title{
Typische Wetterlagen und ihre Darstellung im Schweizer Weltatlas
}

\begin{abstract}
Vorbemerkung
Neben der Seite 20 mit den typischen Wetterlagen zeigt ein instruktives LANDSAT-Bild der Tessiner Landschaft, wie die Atmosphäre über der stark industrialisierten Poebene durch menschliche Einwirkung an Durchsichtigkeit einbüßte. In gleichem Maße wird auch die horizontale Sichtweite mehr und mehr beeinträchtigt, was sich auch im Mittelland in einer kleineren Zahl von Tagen mit Alpensicht bemerkbar macht.
\end{abstract}

\section{Zur Interpretation der NOAA- und METEOSAT-Bilder}

Die typischen Wetterlagen im Atlas werden auf der Grundlage von großräumigen Satellitenbildern präsentiert, vier NOAA-Bilder aus $1450 \mathrm{~km}$ Höhe und zwei METEOSAT-Aufnahmen aus $36000 \mathrm{~km}$ Höhe, wie sie vom Fernsehen her bekannt sind. Mit einer Ausnahme handelt es sich um Abbildungen im sichtbaren Spektralbereich. Für die Föhnlage hingegen wurde der langwellige Infrarotkanal ausgewählt, der den Meteorologen ebenfalls zur Verfügung steht. Infrarotaufnahmen können auch nachts erstellt werden, wenn der sichtbare Bereich ausfällt.

Die uns vertrauten Bilder aus dem sichtbaren Spektralbereich sind ohne weitere Erläuterungen lesbar. Allerdings ist zu beachten, daß weiße Flecken sowohl Wolken als auch Schnee bedeuten können. Im Beispiel «Hochdruckgebiet», in dem die Niederungen beidseits der Alpen von ausgedehnten Nebel- und Hochnebelfeldern bedeckt sind, weist der Winterschnee im Alpenraum eine wesentlich höhere Reflexion (Albedo) auf als die Wolkendecke. Im Normalbild weisen Wolken nur geringe Helligkeitsunterschiede auf; sie sind unabhängig von der Höhenlage alle praktisch weiß. Einen gewissen Einfluß haben die Dicke und die Dichte der Wolken: dünne Wolken erscheinen oft transparent und eher gräulich, mächtige Wolken (z. B. Gewitterzellen) erscheinen blendend weiß. Die feste Erdoberfläche und besonders das Meer sind dagegen dunkel. Sie verschlucken einen größeren Teil der auffallenden Sonnenstrahlung. Einzig die Wüstengebiete zeichnen sich als relativ hell ab.

Im Beispiel «Föhnlage» zeichnet das Infrarotbild die Wärmestrahlung auf, welche von der Temperatur der ausstrahlenden Körper abhängig ist. Da die Temperatur in der Atmosphäre mit der Höhe abnimmt, ausgenommen bei winterlichen Inversionslagen, strahlen die kälte- ren Wolken gegenüber dem wärmeren Erdboden weniger Wärme ab. Sie würden somit auf dem Bild im Gegensatz zu normalen Bildern dunkler erscheinen als der Erdboden. Damit man sich bei der Interpretation nicht umgewöhnen muß, verwendet man bei Infrarotbildern das Negativ. Auf ihm ist das in unseren Breiten ganzjährig ziemlich warme Meer verhältnismäßig dunkel und die feste Erdoberfläche ebenfalls, so daß sich nur geringe Unterschiede ergeben. Bei den Wolken ist dagegen entscheidend, in welcher Höhenlage sie sich befinden. In den obersten Schichten der Troposphäre, in $10-12 \mathrm{~km}$ Höhe, wo die Cirrus- und Cirrostratuswolken feine Eiskristallschleier bilden, herrschen Temperaturen von etwa $-50^{\circ} \mathrm{C}$. Diese Wolken bilden sich im Negativ blendend weiß ab, weil sie wenig Wärmestrahlung abgeben. Tiefere Wolken in der Nähe der Erdoberfläche erscheinen demgegenüber grau. Dieser Unterschied wird im Vergleich der Beispiele "Föhnlage» und "Westwindlage» deutlich. In beiden findet sich über Mitteleuropa eine dicke, bis in die höchsten Schichten hinaufreichende Wolkenmasse, aus welcher im blau schraffierten Gebiet Niederschläge fallen. Bei der Föhnlage erkennt man im Hochdruckgebiet über Osteuropa graue Hochnebelfelder, welche zum Schlechtwettergebiet einen wesentlich größeren Kontrast bilden als im normalen Bild der Westwindlage, wo über Ungarn in einem Hochdruckkeil ebenfalls tiefliegende Wolkenfelder auftraten, jedoch nicht grau, sondern ebenfalls weiß erscheinen. Die hohen, meist verhältnismäßig dünnen Wolken werden daher im Infrarotbild überbewertet. So ist z. B. die scharfe Wolkenkante bei Wien im Föhnbild nicht so bedeutsam, wie sie in dieser Nachtaufnahme erscheint. Man gibt deshalb der Aufnahme im sichtbaren Spektralbereich meist den Vorzug. Für den Meteorologen ist jedoch die 24stündige, ununterbrochene Verfolgung der Wetterentwicklung wichtig.

\section{Allgemeines}

Nach diesen einleitenden Bemerkungen werden im folgenden die sechs Wetterlagen auf S. 20 des Atlasses besprochen. In der Vielfalt der Wettersituationen im Laufe eines Jahres lassen sich zwei Hauptgruppen unterschei-

Max Schüepp, Prof. Dr., em. Dozent der Universität Zürich, Bürglistraße 16, 8304 Wallisellen 
den. Es sind dies einerseits die Lagen mit stärkeren Winden, wie sie vor allem in der kälteren Jahreszeit auftreten, wenn infolge der Polarnacht zwischen Äquator und Nordpol ein starkes Temperaturgefälle auftritt. Sie werden als advektive Lagen bezeichnet und je nach der über dem Alpengebiet vorherrschenden Windrichtung in vier Fälle unterteilt: Südwind mit Föhn nördlich der Alpen, Westwind, Nordwind mit Staulage am Alpennordrand und Ostwind oder Bisenlage. Neben diesen advektiven Lagen mit Luftzufuhr aus anderen Gebieten treten andererseits vor allem in der warmen Jahreszeit, mit ihren geringeren Temperaturunterschieden zwischen Äquator und Pol, Fälle auf, bei denen der Luftmassenaustausch zwischen verschiedenen Gebieten zurücktritt gegenüber dem Austausch zwischen tieferen und höheren Schichten. Sie werden als konvektive Lagen bezeichnet (beide Karten unten auf S. 20). Im engeren Sinne wird mit Konvektion meistens das Aufsteigen der Luft in überhitzten Gebieten bezeichnet, welches zur sommerlichen Haufenwolkenbildung, zu Cumulus- und Cumulonimbusbewölkung führt. Diesem aufsteigenden Ast der Zirkulation ist aber auch eine leicht absinkende Strömung im Umfeld der Wolken beigesellt, was eine Verteilung der von der Sonne eingestrahlten Wärme auf die ganze Luftschicht bewirkt. Als Gegenbewegung zur sommerlichen Konvektion tritt im zentralen Teil der Hochdruckgebiete ein langsames Absinken der Luft, verbunden mit einer Auflösung der Wolken, auf. Zur Darstellung dieser beiden Lagen mit vertikalen, konvektiven Strömungen und mit den zugehörigen geringen horizontalen Druckgradienten (außer in Gewitterböen) sowie langsamen Luftmassenverlagerungen werden keine großräumigen Karten benötigt. Die größere Auflösung ist dagegen von Vorteil, um den bedeutenden Einfluß der regionalen Geländestrukturen zu zeigen. Der Bedeutung der vertikalen Temperaturschichtung in der Atmosphäre in den beiden Fällen «sommerliche flache Tiefdrucklage» und «winterliche Hochdrucklage» wurde mit der Angabe der Temperaturkurve bis auf $5 \mathrm{~km}$ Höhe Rechnung getragen.

In der Kartenserie fehlt eine Lage, in welcher das Zentrum eines Tiefdruckwirbels mit starken horizontalen und vertikal aufsteigenden Strömungen über Mitteleuropa hinzieht. In unseren Breiten sind wir glücklicherweise von den gewaltigen Wirbelstürmen, den Hurrikanen und Taifunen, verschont. Im Alpengebiet ist ein starker Wirbel, wie er in der Karte "Westwindlage» über der Nordsee sichtbar ist, sehr selten. Weil die Alpen wie eine Scheidewand wirken, muß er sich in einen nördlichen und einen südlichen Wirbel aufspalten, wodurch eine großräumige Verwirbelung verhindert und die Sturmenergie durch Reibung stark vermindert wird. Eine Ausnahme von dieser Regel war der gewaltige Weststurm «Vivian» vom 27./ 28.2.1990, indem ein Randtief mit zusätzlichen Wirbeln neben sehr starken horizontalen auch bedeutende vertikale Windströmungen auslöste und in vielen Bergtälern enorme Waldschäden anrichtete.

Die Karten erlauben im Gegensatz zur auf den Sammelbegriff Störungen reduzierten Berichterstattung im Fernsehen eine eingehendere Analyse. Dazu ist über dem
Satellitenbild in den vier Karten mit Advektionslagen der auf Meereshöhe reduzierte Luftdruck mit Isobaren von $5 \mathrm{zu} 5 \mathrm{hPa}$ (Hektopascal, neuere Bezeichnung für Millibar) eingezeichnet, zusammen mit den Fronten nach dem üblichen Auswerteschema von BJERKNES. Dieses schließt zwischen der Warm- und Kaltfront den Warmsektor einer Zyklone ein, welcher dann im Laufe der Entwicklung, wenn die rascher vorrückende Kaltfront (blau) die Warmfront (rot) eingeholt und die Warmluft in die oberen Luftschichten abgedrängt hat, in eine Okklusion (violett) mündet. Die farbigen Pfeile geben die Strömungsrichtung und -geschwindigkeit in der unteren Atmosphärenschicht wieder, den sogenannten geostrophischen Wind, wie er etwa ab $500 \mathrm{~m}$ über Boden in Richtung der Isobaren weht. In Bodennähe erfolgt infolge der Reibung eine Verminderung der Geschwindigkeit und eine Ablenkung der Strömung zum tieferen Luftdruck hin. Diese beträgt etwa $45^{\circ}$, kann aber bei sehr stark profiliertem Gelände noch wesentlich größer sein, bis beinahe eine Strömung in Richtung des Druckgefälles, senkrecht zu den Isobaren, entsteht, so z. B. in den Föhntälern der Alpen. Für die Verlagerung der Fronten ist aber der Wind oberhalb dieser Bodenreibungsschicht maßgebend, der aus der Karte durch die Richtung der Isobaren und ihren Abstand voneinander zu entnehmen ist. Je mehr sich die Isobaren drängen, desto stärker weht der Wind. Bei den beiden Karten zu den konvektiven Lagen mit flachem Tief und Hoch finden wir innerhalb des Gebietes als Folge der geringen Druckgegensätze nur je eine einzige Isobare. Die Serien von schwarzen Pfeilen geben Aufschluß über die Windströmung in etwa $5500 \mathrm{~m}$ Höhe, welche für eine genauere Analyse von Bedeutung ist (vgl. Abschnitt weiter unten über die Rekonstruktion der Höhenwetterkarten).

Wie die Luftdruckkarten für den Januar und den Juli auf S. 160/161 des Atlasses zeigen, befindet sich die Schweiz im Sommer, zur Zeit des geringsten Temperaturgegensatzes zwischen Äquator und Nordpol, am Ostrand einer maritimen Hochdruckzone mit dem Kern über den Azoren. Diese schwächt sich im Winter ab und verlagert sich nach Südosten, so daß - wie die Windpfeile auf S. 160 zeigen - die über dem nördlichen Atlantik herrschenden kräftigen WSW-Winde vielfach auch nach Mitteleuropa eindringen können.

\section{Konvektive und hochdruckbestimmte Wetterlagen}

Wir beginnen die Jahresübersicht im Sommer mit dem Beispiel einer Gewitterlage im August (Karte unten links). Die Luftdruckgradienten sind dort im flachen Tief schwach und demzufolge auch die Winde in Bodennähe. Mit Werten um 1005 bis $1010 \mathrm{hPa}$ auf Meeresniveau liegt der Druck etwas unter dem normalen Sommermittel von $1015 \mathrm{hPa}$ (siehe Karte S. 161). Der lange Pfeil für den Wind in $5500 \mathrm{~m}$ Höhe und die weißen Wolkenfelder zeigen an, daß die Wetterlage nicht stabil ist. Das Temperaturdiagramm weist darauf hin, daß die untere, tagsüber stark erhitzte Luftschicht aufsteigt und Haufenwolken, in 
großen Höhen sogar Gewitterwolken (Cumulonimbuswolken) bildet. Man beachte die blauen Gewittersignaturen. Aus dem südfranzösischen Bergland des Languedoc quillt eine gewaltige Gewittermasse in die obere Troposphäre, wo sie von den sehr starken Höhenwinden mit ihren Cirruswolken mit etwa $80 \mathrm{~km} / \mathrm{h}$ bis in die Westschweiz verschleppt wird. Die Wolkenobergrenze zeigt eine leichte Struktur, soweit diese nicht durch die sich pilzförmig ausbreitenden Cirruswolken verdeckt wird. Im Gegenbeispiel mit dem flachen Hochdruckgebiet (Karte unten rechts) erkennen wir den verschneiten $\mathrm{Al}$ penbogen im Dezember 1982. Nördlich und südlich davon liegen über den Niederungen, besonders über Bayern, der Poebene und westlich des Juras, verbreitet Nebel- und Hochnebelfelder. Die Wolkenobergrenze ist glatt. Ganz anders ist die Temperaturverteilung in dieser Jahreszeit. Die Luft oberhalb der Nebelfelder ist verhältnismäßig trocken und in langsamem Absinken begriffen. Sie erwärmt sich dabei um $1 \% 100 \mathrm{~m}$. Im Nebelgebiet der Niederungen ist es dagegen kalt. Damit bildet sich an der Obergrenze der Nebelschicht ein scharf abgegrenzter Bereich, in dem die Temperatur mit der Höhe zunimmt, eine sogenannte Inversionsschicht. Das Beispiel zeigt ein typisches winterliches Hoch. Solche Hochdrucklagen können aber auch in der warmen Jahreszeit auftreten, erzeugen dann aber nicht mehr ganztägige, sondern nur noch nächtliche Bodenkaltluftschichten, die gelegentlich frühmorgens zu Nebel in den Niederungen führen. Tagsüber können sich dann kleine Haufenwolken ohne große Höhenerstreckung bilden, welche die Sicht nicht nur in den Niederungen, sondern auch im Bergland trüben. Charakteristisch für die Hochdrucklage ist in allen Jahreszeiten das fast völlige Fehlen von Niederschlägen.

Bei beiden Karten wurden für vier Stationen zahlenmäBige Angaben beigefügt. $D a$ bei beiden Wetterlagen die vertikale Temperaturschichtung eine wesentliche Rolle spielt, wurden alpine Stationen in verschiedener Höhenlage ausgewählt. Die Walliser Talstation Sitten und die Hangstation Montana bilden ein Paar mit etwa 1000 m Höhenunterschied (siehe Karte auf S. 3). Die Engadiner Talstation Samaden und die Bergstation der Corvatschbahn ergeben das andere Paar mit $1600 \mathrm{~m}$ Höhendifferenz. Beim flachen Tief errechnet sich aus den Angaben bei beiden Paaren eine Temperaturabnahme von $0,6-0,8^{\circ}$ pro $100 \mathrm{~m}$. Die Feuchtigkeiten weisen allgemein mittlere Werte auf. Ganz andere Verhältnisse finden wir bei der winterlichen Hochdrucklage. Die aufgeführten Morgenwerte geben besonders im Engadin, aber auch in anderen höhergelegenen Alpentälern mit flachen Becken, wie

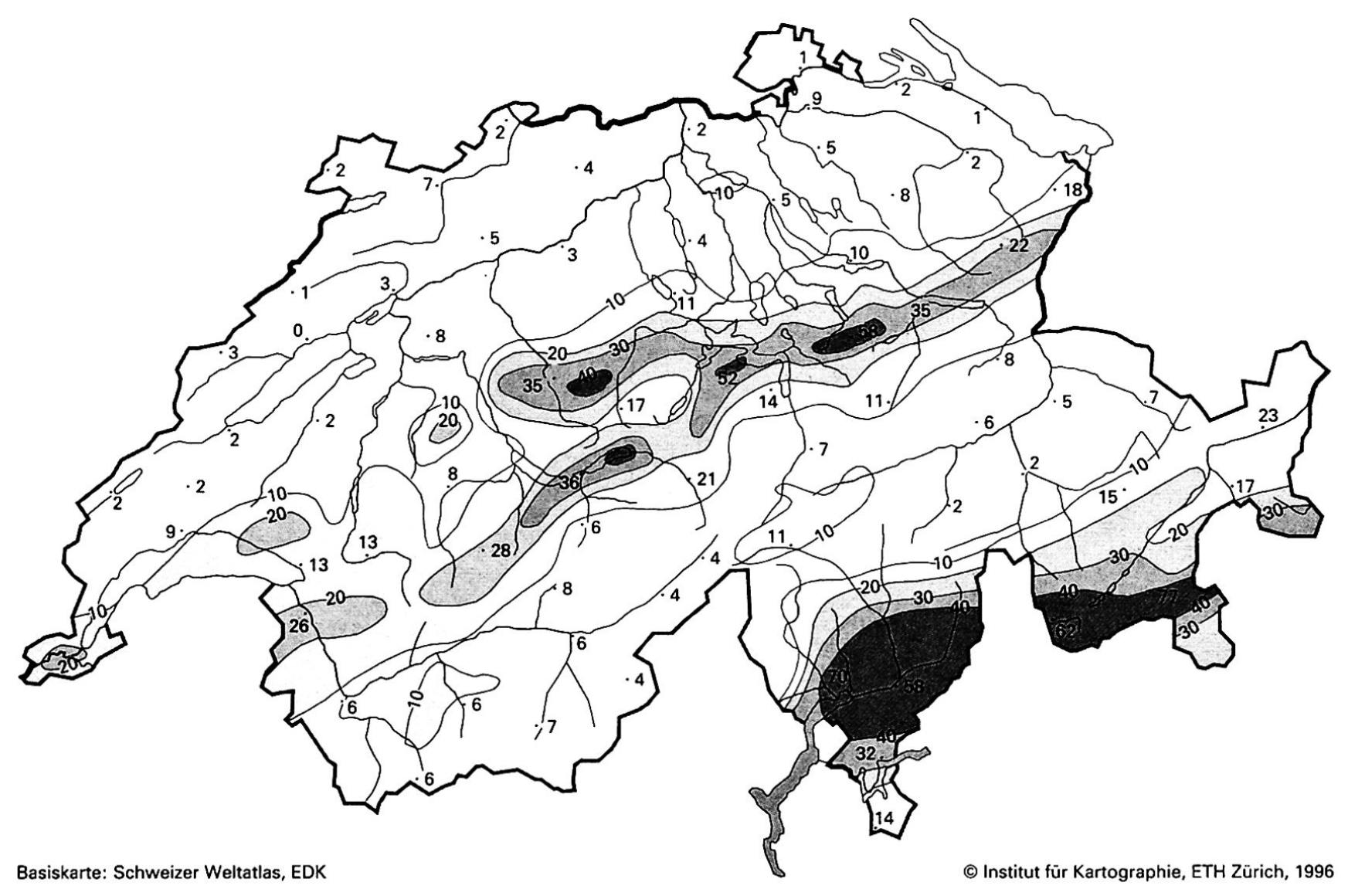

Abb.1 24stündige Niederschläge in einer Gewitterlage am 18.8.1986. 
z. B. in Andermatt oder in La Brévine, extreme Verhältnisse wieder. Sitten ist nun nicht wärmer, sondern $2^{\circ} \mathrm{käl}-$ ter als Montana; zugleich ist es im Talgebiet wesentlich feuchter. Samaden ist nicht mehr $2^{\circ}$, sondern volle $11^{\circ} \mathrm{käl}$ ter als Montana und ebenfalls feucht. Auf dem Corvatsch ist es extrem trocken und nur $2^{\circ}$ kälter als im $2800 \mathrm{~m}$ tiefer gelegenen Sitten, während beim sommerlichen Tief die Temperaturdifferenz $20^{\circ}$ betrug!

Bei den Angaben zum Wind soll im Sommer bei Samaden auf die kräftige SW-Strömung, auf den sogenannten Malojawind, hingewiesen werden. Es handelt sich um einen bei sonnigem Wetter auftretenden, aus dem Comerseegebiet aufsteigenden Talwind, der über den flachen Malojapaß hinüberweht und die sommerliche Hitze mildert, oft aber auch unangenehm stark sein kann.

Während bei der Hochdrucklage keine Niederschläge auftreten, finden wir in der mit NS bezeichneten Kolonne überall Niederschlag aus diesen Gewitterregen, jedoch örtlich von unterschiedlicher Menge. Als Ergänzung zeigt die Abb. 1 eine Übersicht über die 24stündigen Niederschlagsmengen auf allen Regenmeßstationen. Man erkennt, wie bei den meisten Wetterlagen, eine großräumige Anordnung der gefallenen Mengen. In diesem Falle lassen sich zwei Niederschlagszonen längs des nördlichen und des südlichen Alpenrandes erkennen, jedoch kleinräumig mit großen Unterschieden von Ort zu Ort, sowohl mengenmäßig als auch bezüglich des Einsetzens des Regens, wie das für Gewitterniederschläge bei konvektiven Lagen charakteristisch ist. Im Kanton Genf lagen die Mengen zwischen 10 und $28 \mathrm{~mm}$. Der Regen setzte in Genf Cointrin um $13 \mathrm{~h} 25$ ein, in Interlaken erst um $15 \mathrm{~h} 40$ mit $36 \mathrm{~mm}$, während Thun nur $5 \mathrm{~mm}$ erhielt. Auch südlich der Alpen finden wir diese großen Unterschiede: in Locarno fielen $70 \mathrm{~mm}$ von $15 \mathrm{~h}$ an, während $12 \mathrm{~km}$ westlicher Mosogno nur $3 \mathrm{~mm}$ erhielt, obwohl es mit 412 mm während der Föhnlage vom 10.9.1983 zu den Stationen mit den absolut größten Tagesmengen gehört.

\section{Advektive Wetterlagen, allgemeiner Überblick}

Eine Advektion oder Luftzufuhr nicht durch vertikalen Austausch, sondern durch mehr oder weniger horizontale Strömungen ist notwendig zum Ausgleich der Temperaturunterschiede zwischen den äquatorialen und den polaren Gebieten. Dieser Austausch kann entweder durch Verwirbelung der Luftschichten an der Grenze zwischen Warm und Kalt geschehen, durch die Bildung von Tiefdruckwirbeln an der Kalt-Warm-Grenze, der sogenannten Polarfront, oder durch großräumige Vorstöße von Warmluft Richtung Pol, in einem andern Sektor der Erdhalbkugel mit einem polaren Kälteausbruch Richtung Äquator gekoppelt. Eine erste, weniger wirksame Form des Austausches mit einer allgemeinen Westströmung, in der sich einzelne Wirbel bilden, wird als Zonalzirkulation bezeichnet. Diese Westwindlage (Karte oben rechts) stellt sich häufig während des Winterhalbjahres ein, flaut dann im Mai ab, erscheint aber aufs neue im Frühsommer, wenn das Kältereservoir des Polargebietes durch den nun ständigen Sonnenschein am Pol abgebaut wird und bei uns die Sommerhitze einsetzt. Die beiden miteinander gekoppelten Ausbrüche von Warmluft vom Äquator gegen den Pol mit einer allgemeinen Südströmung und die Nordströmung des polaren Kaltluftausbruchs bilden die beiden Äste der sogenannten Meridianzirkulation (Karten links oben und Mitte links). Diese ist wesentlich wirksamer für den Temperaturausgleich zwischen Äquator und Pol. Sie stellt sich deshalb vor allem im Winterhalbjahr ein. Anfänglich gibt es mit den Novemberstürmen noch häufig Zonalzirkulation. Dann aber genügt die Verwirbelung in der Westströmung nicht mehr zum Ausgleich, und es kommt zu großräumigen Wellenbildungen mit entsprechenden Süd- und Nordwinden. Da diese Wellen nicht an eine bestimmte geographische Länge gebunden sind, können die Warm- und Kaltluftvorstöße sowohl über Europa als auch über Asien oder Amerika erfolgen, wobei aber die großen Gebirgszüge, speziell das tibetische Hochland und die Rocky Mountains, steuernd wirken. Bei der Südströmung entsteht nördlich der Alpen eine Föhnströmung in den Quertälern, vor allem im Frühjahr und Herbst, etwas weniger häufig im Hochwinter, weil dann die im Mittelland lagernde Kaltluft in den Niederungen hemmend wirkt. Die Nordströmung, welche auf der Alpennordseite Staulage mit starker Bewölkung und Niederschlägen bringt, stellt sich vorwiegend in der zweiten Winterhälfte ein. Dann hat der Alpensüdhang heiteren Himmel mit Nordföhn, während im Rhonetal südlich von Lyon der Mistral weht. Im Kartenbild (Mitte links) erkennt man die Schönwetterzone in der Poebene und im nördlichen Teil des italienischen Stiefels.

Bei großen Kaltlufteinbrüchen verlagert sich die Verwirbelung der Luftmassen in den Mittelmeerraum, wie es die Karte Mitte rechts mit der Bisenlage zeigt. Über Europa, das von einer großen, aus dem nordrussischen Raum eingebrochenen Kaltluftmasse bedeckt ist, hat sich ein umfangreiches Hoch über der Ostsee aufgebaut, welches die sonst in den gemäßigten Breiten vorherrschenden Westwinde verdrängt hat. Man spricht in diesem Fall von einer Blockierung. Sie tritt vor allem im Januar und $\mathrm{Fe}$ bruar auf, wie die Häufigkeitsverteilung der Bise in Abb. 2 zeigt. An der norddalmatinischen Küste ergießt sich in der Karte der Bisenlage die kalte kontinentale Luft mit einer kräftigen Bora über die Adria und verursacht Aufhellungen beidseits der istrischen Halbinsel.

\section{Westwindlage}

In der Karte der Westwindlage ist das Frontensystem der Nordseezyklone am schönsten erkennbar, weil das Tief im Höhepunkt seiner Entwicklung noch einen sehr deutlichen Gegensatz zwischen der im Warmsektor mitgeführten Warmluft (roter Pfeil über Frankreich) und den beiden Kaltluftgebieten (blaue Pfeile über Berlin und 


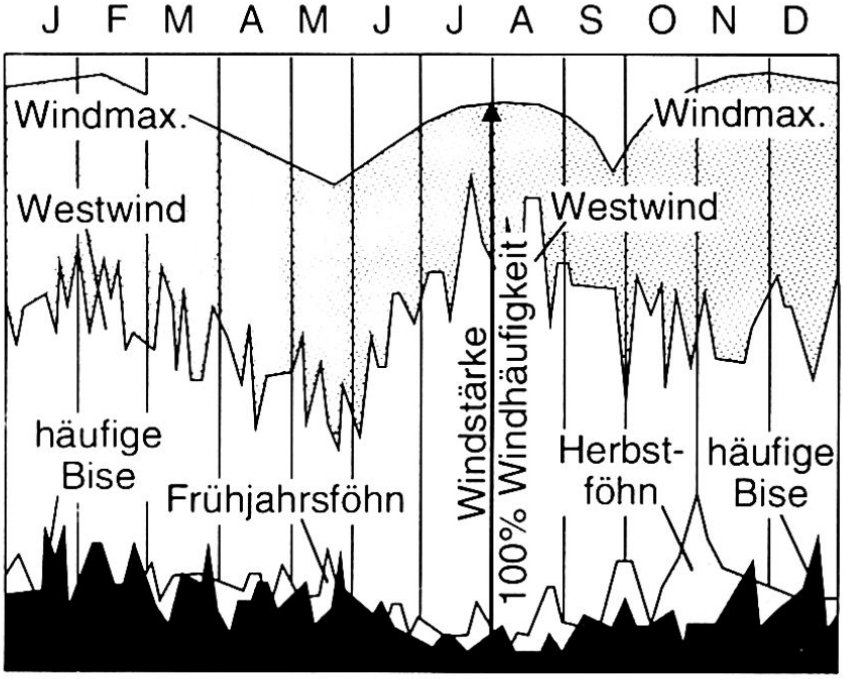

Abb.2 Windhäufigkeiten auf dem Säntis im Durchschnitt der Jahre 1883-1940 (SCHÜEPP 1950).

nordwestlich der Bretagne) vor der rot eingezeichneten Warmfront (Nordsee-Montreux) und hinter der blauen Kaltfront (Holland-Bretagne) zeigt. Über Deutschland liegt ein breites Gebiet mit geschlossener Bewölkung, welche sich nach Westen hin allmählich verdichtet und in ein großes Niederschlagsgebiet mit Südwestwind übergeht. Dann folgt der Warmsektor mit Westwind und meist geschlossener Bewölkung, die nur gegen Süden hin von einzelnen Aufhellungen unterbrochen ist. Hinter der Kaltfront, welche mit starken, böigen Winden einbrechen kann, dreht der Wind auf Nordwest. Den frontalen Regenschauern folgt ein starkes Aufreißen der Bewölkung im Kanalgebiet zwischen Frankreich und Südengland und dahinter über dem Atlantik wieder einsetzende zellenförmige Bewölkung mit Cumuli. Die Isobaren sind allgemein dicht gedrängt, die Winde dementsprechend kräftig. Die schwarzen Höhenwindpfeile zeigen, daß das ganze Tiefdruckgebiet rasch nach ENE weiterwandert. Es wird nicht das letzte einer Wirbelserie sein. Am Westrand der Karte geht die Kaltfront bereits wieder in eine neue Warmfront über. Die Wettermeldungen von Basel und Zürich in der Kartenlegende beschreiben den in der Karte festgehaltenen Zustand um $13 \mathrm{Uhr}$ in seinem zeitlichen Ablauf beim Durchzug der Zyklone mit den entsprechenden Temperatur-, Bewölkungs- und Niederschlagswerten.

\section{Föhnlage}

Das ausgewählte Beispiel entspricht nicht einer durchschnittlichen Föhnlage, sondern in bezug auf die Luftdruck- und Strömungsverhältnisse in den unteren Schichten einem extremen Fall. Die zum Teil verheerenden Folgen trugen ihm den Beinamen «Jahrhundertföhn» ein. Der Tiefdruckwirbel liegt nicht über der Nord- see wie bei der Westwindlage, sondern über dem Atlantik. Er ist nicht mehr so jung und lebenskräftig wie jener, auch wenn er größer und tiefer ist. Die lange, violette Frontschleife, die vom Zentrum aus über Südengland nach Ostfrankreich verläuft, ist eine Okklusion. Sie entsteht, wenn die rasch vorrückende Kaltfront die Warmfront einholt und die beiden Niederschlagsgebiete in ein einziges verschmelzen. Im Mittelmeerraum finden wir rund um Algier noch den Warmsektor, diesmal mit geringer Bewölkung, und die Warmfront zwischen Korsika und Sardinien. Die von dort bis ins Rheintal eingezeichnete gestrichelte Linie soll andeuten, daß die von Süden anströmende Luft durch die über dem Golf von Genua und der Poebene lagernde Kaltluft in die Höhe abgedrängt wurde. Sie gelangt erst nördlich des Alpenkammes wieder als Föhn in Bodennähe. Auf der Alpensüdseite ist der für diese Wetterlage typische Niederschlag eingezeichnet. Im Normalfall reißt mit dem Föhn die Bewölkung im Lee auf der Alpennordseite auf und bildet das bekannte blaue Föhnfenster. Das ist in diesem Beispiel nicht der Fall, weil der Föhn am 7./8. November 1982 in der Höhe ungemein stark war und den Alpenkamm gewissermaßen überschoß, dafür aber im Mittelland und sogar im südlichen Deutschland Fuß faßte. Dabei verschob sich der Scheitelpunkt der Strömung vom Alpenkamm gegen Norden, so daß die sich vom Westen langsam heranschiebende hohe Bewölkung der Störungszone (Okklusion) dicht blieb und nur zeitweise kleine Föhnlücken entstehen konnten. Bei dieser Lage erhalten in einzelnen Fällen die südlichen, hinteren Teile der Föhntäler sogar etwas Niederschlag; die Luft ist nach Glarnerdialekt «dimmrig», d. h. getrübt statt klar. Man spricht dann von einem Dimmerföhn.

Andererseits ist aus dem Beispiel ersichtlich, wie der (schraffiert dargestellte) Niederschlag vor der Okklusion über Nordostfrankreich durch die absinkende Föhnluft unterdrückt wurde. Jeder Föhn hat sein eigenes Gesicht! Die vier aufgeführten Schweizer Stationen schildern die Verhältnisse in Kürze: Temperaturdifferenz zwischen Basel und Altdorf $10^{\circ}$, Feuchtigkeit im Föhntal nur 30\%, am Alpensüdhang in Lugano verhältnismäßig kalt, Regen und und leichter Nordwind, auf dem Gütsch ob Andermatt SSE-Sturm, Erwärmung der Luft um $19^{\circ}$ beim Absteigen zum $1838 \mathrm{~m}$ tiefer gelegenen Altdorf, d.h. etwa $1 \% 100 \mathrm{~m}$.

\section{Nordlage oder Staulage}

Im Beispiel der Staulage mit NNW-Strömung und Kaltluftzufuhr von der Nordsee her wurde bereits auf den Nordföhn und den Mistral im Rhonetal (blaue Pfeile bei Mailand und bei der Rhonemündung) hingewiesen. Hier sind keine komplizierten Frontensysteme in der Nähe. In direkter Linie stößt die über der Nordsee durch die typische flockenförmige Haufenbewölkung erkennbare Kaltluft zu den Alpen vor. Sie wird zwar durch die Vogesen, den Schwarzwald und den Jura etwas gebremst und nimmt in den tieferen Lagen den Umweg über Lyon und 
das Rhonetal, überquert aber zur Hauptsache die Alpen. Sie bringt daher bei allen drei aufgeführten Stationen Basel, Einsiedeln und Gütsch - Schneefälle, an Menge gegen den Alpenkamm zunehmend, entsprechend der verstärkten Hebung und Abkühlung. Dann folgt der Abstieg in die Tessiner Täler, wo Sonnenschein und klare Luft die Folge sind. Dank der zusätzlichen Wärme durch die Sonnenstrahlung ist die Luft in Lugano, das $2000 \mathrm{~m}$ tiefer liegt als der Gütsch, sogar $23^{\circ}$ wärmer (überadiabatischer Temperaturgradient von $1,14^{\circ} / 100 \mathrm{~m}$ ) und mit $30 \%$ Lufteuchtigkeit sehr trocken, wie im Beispiel des Südföhns in Altdorf.

\section{Bisenlage}

Auch im Beispiel der Bisenlage fehlen die Fronten im europäischen Raum. Nur im Mittelmeer östlich von Sizilien finden wir eine Kaltfront mit der nachfolgenden, charakteristischen aufgebrochenen Bewölkung und vereinzelten Schneefällen in Sardinien und Korsika. Auch in den Ostalpen bei Wien und über Ungarn und Kroatien gibt es Schneefälle. Aber sie sind von geringer Bedeutung bei den tiefen Temperaturen der von Osten heranströmenden Kaltluft. Die ENE-Strömung hat in Bern nur eine geringe Geschwindigkeit $(7 \mathrm{~km} / \mathrm{h})$. In Genf, wo Jura und Alpen trichterförmig zusammenlaufen, ergeben sich bereits $24 \mathrm{~km} / \mathrm{h}$, auf den Jurahöhen in La Chaux-de-Fonds $30 \mathrm{~km} / \mathrm{h}$ und auf La Dôle bei $-12^{\circ} \operatorname{sogar} 78 \mathrm{~km} / \mathrm{h}$, somit nicht die angenehmsten Verhältnisse trotz des blauen Himmels.

\section{Rekonstruktion der Höhenwetterkarten}

Die Höhenwetterkarte im 500-hPa-Niveau stellt für den Meteorologen - neben der Bodenwetterkarte und der Karte im 850-hPa-Niveau (auf etwa 1500 m Höhe) - ein wesentliches Hilfsmittel zur Beurteilung der Wetterlage dar. Sie ist auch, weil sie ungefähr in der Mitte der Troposphäre liegt, für die numerische Berechnung der Prognosekarten wichtig. Die schwarzen Windpfeile in den vier Advektionskarten sind eine Grundlage für die Konstruktion der zugehörigen Höhenwetterkarte, denn sie wurden längs den Höhenkurven der 500-hPa-Fläche eingezeichnet. Die Äquidistanz beträgt $200 \mathrm{~m}$.

Auf einem Transparentpapier können wir beispielsweise diese Kurven für die Föhnlage rekonstruieren. Wir starten südlich von Gibraltar und ziehen die Kurve zu den Pfeilen bei den Balearen, bei Zürich und weiter nach NNE bis südlich von Stockholm, wo sie nach rechts umbiegt und über Polen ins östliche Mittelmeer verläuft. Eine zweite Kurve verläuft vom Atlantik nach Madrid und dann ebenfalls nach NNE östlich an London vorbei zur Nordsee. Eine dritte geschlossene elliptische Kurve umschließt das Tiefdruckgebiet auf dem Atlantik, womit diese Karte bereits aufgezeichnet ist.

Was läßt sich nun dem Kartenpaar Bodenkarte-Höhenwetterkarte entnehmen? Das Tief über dem Atlantik liegt in der Höhe fast am gleichen Ort wie am Boden. Das bedeutet, daß die Temperaturverteilung um das Zentrum schon sehr ausgeglichen ist. Es handelt sich damit um ein altes Tief, das die "Lehr- und Wanderjahre» schon hinter sich hat und sich nicht mehr rasch bewegt. Das Hoch über dem Balkan ist dagegen in der Höhe viel weiter im Westen anzutreffen, ein Zeichen dafür, daß seine Westseite wesentlich wärmere Luft aufweist als die Ostseite und daß sich ein starker Schub Warmluft aus dem afrikanischen Raum nach Mitteleuropa eingestellt hat. Das Hoch ist somit thermisch asymmetrisch und damit noch in Entwicklung begriffen und bereit abzuwandern. Die Fronten über Mitteleuropa können ostwärts vorstoßen, womit die Föhnlage dann zu Ende gehen wird.

Bei der Westwindlage finden wir vier verschiedene Höhenkurven, welche alle von West nach Ost verlaufen, aber nach Osten stark auseinanderfächern. Über dem Nordsee-Tiefdruckzentrum der Bodenkarte weht ein kräftiger WSW-Wind. Das Tief wird durch die Höhenströmung rasch nach Skandinavien verfrachtet. Auf seiner Ostseite, dort wo die Auffächerung der Höhenkurven im 500-hPaNiveau auftritt, wird der Luftdruck fallen. Zugleich wird sich der Warmsektor des Tiefs verkleinern, es wird altern, und seine rasche Verlagerung wird allmählich abnehmen.

Bei der Staulage läßt sich die Höhenwetterkarte mit drei Kurvenscharen rekonstruieren. Die innerste Kurve verläuft von der Nordsee nach Zürich, biegt dort um und erreicht später Warschau. Die äußerste verläuft im Azorenhoch von NW nach SE. Das Höhentief liegt also über Polen und damit wesentlich weiter im Westen als das Bodentief. Es ist also noch nicht ausgeglichen, sondern wandert noch weiter, was auch durch den bereits sichtbaren Warmsektor eines neuen Wirbels nordwestlich von Irland belegt ist, der den vorangehenden Hochdruckkeil gegen Mitteleuropa abdrängt.

Die Karte mit der Bisenlage weist drei kleinere Kurven auf, worunter eine offene, vom Atlantik über Madrid gegen Algerien weisende. Sie verläuft quer über das flache Bodenhoch, ein Hinweis, daß speziell bei bodennahen östlichen Winden die Höhenwetterkarte oft stark von der Bodenkarte abweichen kann. Das Tief über dem Mittelmeer liegt in der Höhe etwas nördlich des Bodenzentrums, doch ist der Unterschied nicht bedeutend. Die Nordhälfte Italiens weist somit nur wenig tiefere Temperaturen auf als die Südhälfte, weil die Kaltluft das Tief schon umrundet hat, wie aus der flockigen Bewölkung und aus dem flachen, in zwei Zentren aufgespaltenen Zentralbereich des Tiefs hervorgeht. Das Hoch über der Ostsee und Rußland erscheint in der Höhe als kleine Ellipse über Großbritannien und der Nordsee, weit weg vom italienischen Tief der Bodenkarte, so daß die Winde über dem Kontinent in der Höhe verhältnismäßig schwach sind und die ENE-Strömung in den bodennahen Schichten nicht mit der Höhe stärker wird, wie es beim Westwind meistens der Fall ist. Eine rasche Änderung der Wetterlage ist somit nicht zu erwarten, aber eine Milderung der Kälte durch die weitere Alterung des italienischen Tiefs und damit das Nachlassen der ENEStrömung in der Schweiz. 
Diese vier Beispiele zeigen, wie aus der Kombination von Boden- und Höhenwetterkarte eine wesentlich bessere Beurteilung des künftigen Wettergeschehens und ein guter Überblick über die allgemeine Situation möglich sind. Mit der Kenntnis der gestrigen Karten und dem Verfolgen der Bewölkung anhand der Satellitenbilder am Fernsehen ergibt sich eine gute Beurteilungsmöglichkeit, allerdings nicht auf mehrere Tage hinaus. Dafür braucht es Computerberechnungen, welche die Entwicklung für alle Höhenlagen zu beurteilen erlauben. Zusätzliches eigenes Beobachten und Verfolgen des Wettergeschehens, wie z. B. der Zugrichtung der Wolken am Himmelgewölbe, gibt nicht nur zusätzliche Hinweise, sondern steigert die Freude an der Naturbeobachtung, welche für uns moderne Menschen so wichtig ist.

\section{Begleitende Literatur}

Wer sich näher für Wetterabläufe interessiert, dem sei das 1989 in Meyers Lexikonverlag erschienene Buch «Wie funktioniert das? Wetter und Klima» empfohlen. In kurzen, eingehend bebilderten Abschnitten werden die einzelnen Teilbereiche behandelt. Die hier besprochenen, synoptischen Erscheinungen sind auf den S. 138-183 mit vielen farbigen Wolkenbildern dargestellt.

Die Darstellung der Windhäufigkeiten auf dem Säntis findet sich in SCHÜEPP, M. (1950): Wolken / Wind und Wetter. Büchergilde Gutenberg Zürich. 263 S., Fig. 79. Die hier mit einer Karte dargestellte Föhnlage hat im
Buch von Michael Kuhn «Föhnstudien», 1989 herausgegeben von der Wissenschaftlichen Buchgesellschaft Darmstadt, im Beitrag von Karl Frey «Der Jahrhundertföhn vom 8.11.1982» eine eingehende Bearbeitung gefunden. Der gleiche Aufsatz findet sich in der Meteorologischen Rundschau 37, 1984, S. 209-220.

Wer die einzelnen Wettertypen näher untersuchen möchte oder Demonstrationsmaterial in größerem Maßstab benötigt, verfügt mit den 10 Tafeln zu den einzelnen Wetterlagen im Klimaatlas der SMA über das nötige Material. Die 2. Lieferung von 1984 umfaßt zwei Hochdrucklagen, ähnlich wie im Schweizer Weltatlas, die 4. Lieferung von 1987 zwei Flachdrucklagen, die eine mit lokalen Wärmegewittern in einer instabilen Luftmasse, die andere mit Frontgewittern bei Einschüben von kälteren maritimen Luftmassen von Westen her, wobei die einzelnen Gewitterherde je nach Vorbedingungen an verschiedenen Stellen und zu verschiedenen Zeiten auftreten und daher schwer vorhersehbar sind. Die 5. Lieferung enthält weitere 6 Wetterlagen: Tiefdrucklage mit einem Zentrum tiefen Druckes knapp westlich der Schweiz und bedeutenden Niederschlägen beidseits der Alpen, Westwindlage, Staulage auf der Alpennordseite, Ostwindlage mit Bise nördlich der Alpen, zwei Südwindlagen mit Föhn bis ins Alpenvorland hinaus oder nur in den Alpentälern. Die Bogen im Format $34 \times 49 \mathrm{~cm}$ enthalten je 4 Kartendarstellungen: das Satellitenbild Europa, die Boden- und Höhendruckkarte Europas, markante Wettererscheinungen in der Schweiz und die Bodendruckkarte der Schweiz mit regionalen Windströmungen. Die Kartenrückseiten enthalten ausführliche Beschreibungen der Wetterlagen in Deutsch und Französisch, verfaßt von Gian Gensler. 

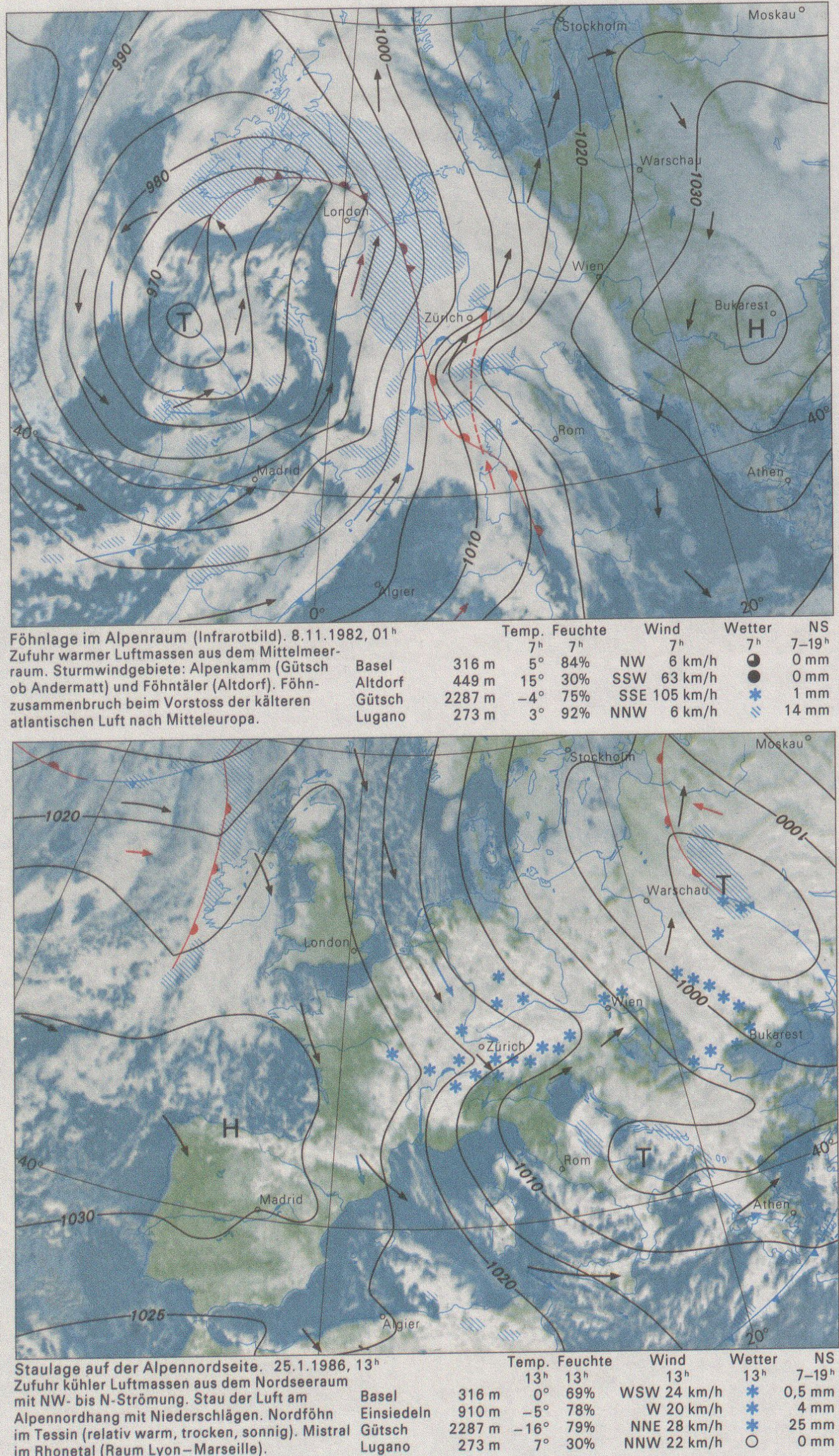

\section{Schweiz, Wetterlagen ( 2 von 6 Karten )}

